\title{
LincRNA-p21 Inhibits Cisplatin-Induced Apoptosis of Human Renal Proximal Tubular Epithelial Cells by Sponging miR-449a
}

\author{
Zhen Li Gang Hou \\ Emergency Department, Zaozhuang Municipal Hospital, Zaozhuang, China
}

\author{
Keywords \\ Cisplatin-induced acute kidney injury · lincRNA-p21 · Renal \\ proximal tubular epithelial cells $\cdot$ miR-449a $\cdot$ Apoptosis
}

Conclusion: Therefore, lincRNA-p21 is downregulated in CIA and may sponge miR-449a to inhibit cisplatin-induced apoptosis of HRPTEpCs. (c) 2021 The Author(s)

Published by S. Karger AG, Basel

\begin{abstract}
Introduction: LincRNA-p21 is predicted to interact with miR$449 a$, which plays a protective role in cisplatin-induced acute kidney injury (CIA). Objective: This study aimed to analyze the involvement of lincRNA-p21 in breast cancer patients with CIA. Methods: Levels of lincRNA-p21 in plasma from $\mathrm{CIA}$, triple negative breast cancer, and control groups were measured by performing RT-qPCR. The potential interaction between lincRNA-p21 and miR-449a was first predicted by RT-qPCR. The relationship between lincRNA-p21 and miR449a was analyzed by overexpression experiment. Results: We found that lincRNA-p21 is downregulated in CIA. Dual luciferase activity assay showed that lincRNA-p21 and miR449a can interact with each other, while overexpression of lincRNA-p21 and miR-449a failed to affect the expression of each other. In human renal proximal tubular epithelial cells (HRPTEpCs), cisplatin led to the upregulated miR-449a but downregulated lincRNA-p21. Interestingly, lincRNA-p21 overexpression led to reduced enhancing effects of miR449a on the cisplatin-induced apoptosis of HRPTEpCs.
\end{abstract}

\section{Introduction}

Since the approval by FDA in 1979, cisplatin has become one of the most commonly used chemotherapy drugs for cancer treatment [1]. Cisplatin can combat multiple types of cancer with different origins, such as blood vessels, bones, muscles, and soft tissues [2]. In the treatment of breast cancer, such as triple negative breast cancer (TNBC), cisplatin is frequently used after surgery to prevent cancer recurrence [3]. Although cisplatin can effectively kill cancer cells in most cases, the long-term application will induce drug resistance and severe adverse effects, such as nephrotoxic $[4,5]$. In effect, the prevention of cisplatin-induced acute kidney injury (CIA) has become one of the focuses of studying the clinical application of cisplatin. However, the underlying mechanism is complicated, and effective preventive approaches are still lacking [6].
(C) 2021 The Author(s)

Published by S. Karger AG, Basel

This article is licensed under the Creative Commons AttributionNonCommercial-NoDerivatives 4.0 International License (CC BYNC-ND) (http://www.karger.com/Services/OpenAccessLicense) Usage and distribution for commercial purposes as well as any distribution of modified material requires written permission.
Correspondence to:

Gang Hou, uk9182@163.com 
It has been reported that the application of cisplatin can globally affect gene expression in human body [7], and some of the differentially expressed genes have been proven to participate in cisplatin-induced side effects [8]. Besides protein coding genes, noncoding RNAs, such as miRNAs and long (>200 nt) noncoding RNAs (lncRNAs), also have critical roles in mediating the function of cisplatin [9]. A recent study reported that miR-449a had enhancing effects on the cisplatin-induced injury in NRK$52 \mathrm{E}$ cells, which are rat kidney cells [10]. However, the role of miR-449a in human kidney injury induced by cisplatin is unknown. LincRNA-p21 is a critical player in several types of cancer [11]. Our bioinformatics analysis revealed the potential interaction between lincRNA-p21 and miR-449a. This study was therefore carried out to investigate the interaction between them in cisplatin-induced CIA in TNBC patients after surgery.

\section{Materials and Methods}

Research Subjects

This study included 60 TNBC patients with CIA (CIA group, age range from 48 to 67 years old, and mean age $58.4 \pm 6.0$ years old), 60 TNBC patients without CIA (TNBC group, age range from 48 to 67 years old, and mean age $58.3 \pm 6.1$ years old), and 60 healthy females (control (C) group, age range from 48 to 67 years old, and mean age $58.3 \pm 5.7$ years old). All participants were enrolled at the Zaozhuang Municipal Hospital between May 2017 and May 2019. This study passed the review of the Ethics Committee of Zaozhuang Municipal Hospital (SD-2017-ZZH348392). All The 120 breast cancer patients were treated by surgical resection of the tumors, and cisplatin was used postoperatively. No significant differences in the use of cisplatin (dosage and treatment duration) were found between 2 patient groups. Patients with acute kidney injury unrelated to the use of cisplatin were excluded. All healthy controls received systemic physiological exam at the physiological health center of aforementioned hospital, and all their physiological functions are normal. All participants signed the informed consent.

\section{Blood Extraction and Plasma Preparations}

Blood ( $5 \mathrm{~mL}$ ) was extracted from each participant under fasting conditions 1 day after admission. Blood was mixed with EDTA, and the mixture was centrifuged at $1,200 \mathrm{~g}$ at room temperature for $10 \mathrm{~min}$ to separate plasma samples. All plasma samples were stored at $-80^{\circ} \mathrm{C}$ before use.

Human Renal Proximal Tubular Epithelial Cells (HRPTEpCs)

All in vitro cell experiments were performed using HRPTEpCs (Sigma-Aldrich). HRPTEpCs were cultivated in a medium composed of $10 \% \mathrm{FBS}$ and $90 \%$ renaEpi growth medium at $37^{\circ} \mathrm{C}$ in a $5 \% \mathrm{CO}_{2}$ incubator with $95 \%$ humidity. Cells were harvested at about $80 \%$ confluence.

\section{Cell Transfections}

Expression vector of lincRNA-p21 and mutant lincRNA-p21 was constructed using pcDNA 3.1 vector as backbone. Negative control
(NC) miRNA, anti-NC and miR-449a mimic, and antagomiR-449a were purchased from Sigma-Aldrich. HRPTEpCs were transfected with either lincRNA-p21 expression vector or miR-449a mimic (40 $\mathrm{nM}$ ) using lipofectamine 2,000 (Invitrogen). Untransfected cells were used as C cells. The NC group was transfected with NC miRNA or empty vector. Cells were harvested at 48 -h post-transfection.

Prediction of Interaction between lincRNA-p21 and miR-449a

The possible base pairs formed by lincRNA-p21 and miR-449a were predicted by IntaRNA 2.0 [12]. The sequence of lincRNAp21 was used as the long sequence and miR-449a sequence was used as the short sequence. All other parameters were default.

\section{Dual Luciferase Activity Assay}

The interaction between lincRNA-p21 and miR-449a was further assessed by performing dual luciferase activity assay. The pGL3 vector (Promega Corporation) was used to construct the luciferase vector of lincRNA-p21 and mutant lincRNA-p21. HRPTEpCs were co-transfected with either the combination of lincRNAp21 vector or mutant lincRNA-p21 and miR-449a mimic (miR449a group) or the combination of lincRNA-p21 vector and NC miRNA (NC group). Luciferase activities were measured at 48-h post-transfection using the Dual-Luciferase Reporter Assay System (Promega Corporation).

\section{RNA Preparations}

Total RNAs were extracted from both plasma samples and HRPTEpCs using TRIzol reagent (Invitrogen). The RNA extraction was performed with the miRNeasy Serum/Plasma kit (Qiagen). All steps were completed following the manufacturer's instructions. RNA samples were precipitated using $85 \%$ ethanol to harvest miRNAs. DNase I (Invitrogen) was used to digest all RNA samples to remove genomic DNAs. In cases of cisplatin treatment, HRPTEpCs were treated with $0,1,2$, or $3 \mu \mathrm{M}$ of cisplatin for $48 \mathrm{~h}$ before use.

\section{$R T-q P C R$}

To measure the expression levels of lincRNA-p21, reverse transcription was carried out using the Bio-Rad iScript cDNA Kit (Bio$\mathrm{Rad})$, and qPCR reactions were prepared using the SYBR Green PCR Kit (Takara Bio). The expression levels of lincRNA-p21 were determined using $18 \mathrm{~S}$ rRNA as the endogenous control. All-in-1 ${ }^{\mathrm{TM}}$ miRNA qRT-PCR Detection Kit (Genecopoeia) was used to measure the expression levels of mature $\mathrm{miR}-449 \mathrm{a}$. Three replicate reactions were set for each experiment, and the relative gene expression levels were normalized using $2^{-\Delta \Delta \mathrm{Ct}}$ method.

\section{Flow Cytometry Analysis}

HRPTEpCs harvested at 48-h post-transfection were subjected to cell apoptosis assay. Cells were cultivated in aforementioned cell culture medium containing $3 \mu \mathrm{M}$ of cisplatin for another $48 \mathrm{~h}$, followed by staining with FITC Annexin V and propidium iodide for 15 min in dark. Apoptotic cells were separated through flow cytometry using BD FACSCanto II system.

\section{Statistical Analysis}

All experiments were performed in 3 biological replicates, and the mean values were used for data analysis. The Kruskal-Wallis test was used for multigroup analysis. Comparisons between 2 groups were performed by the Mann-Whitney test. $p<0.05$ was considered as statistically significant. 


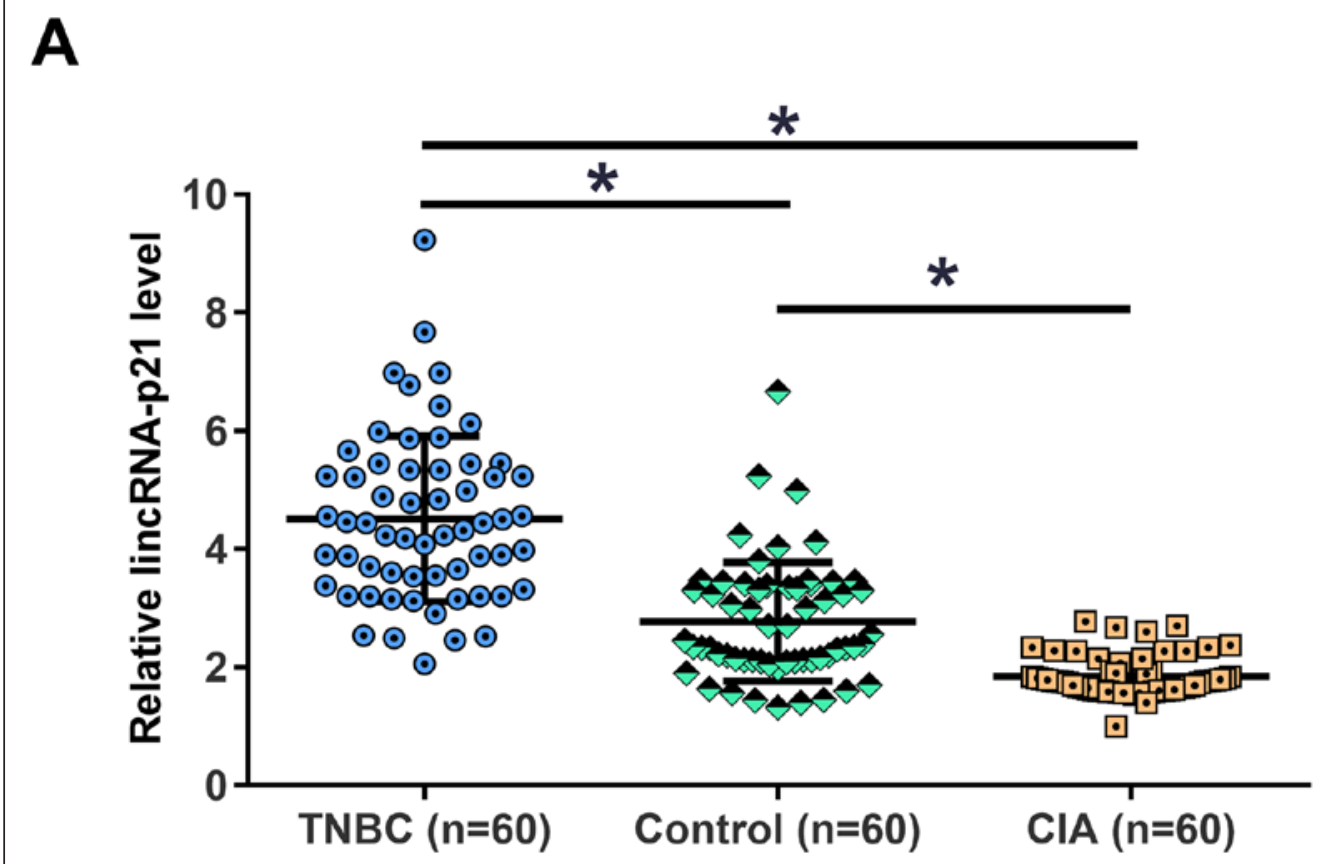

B

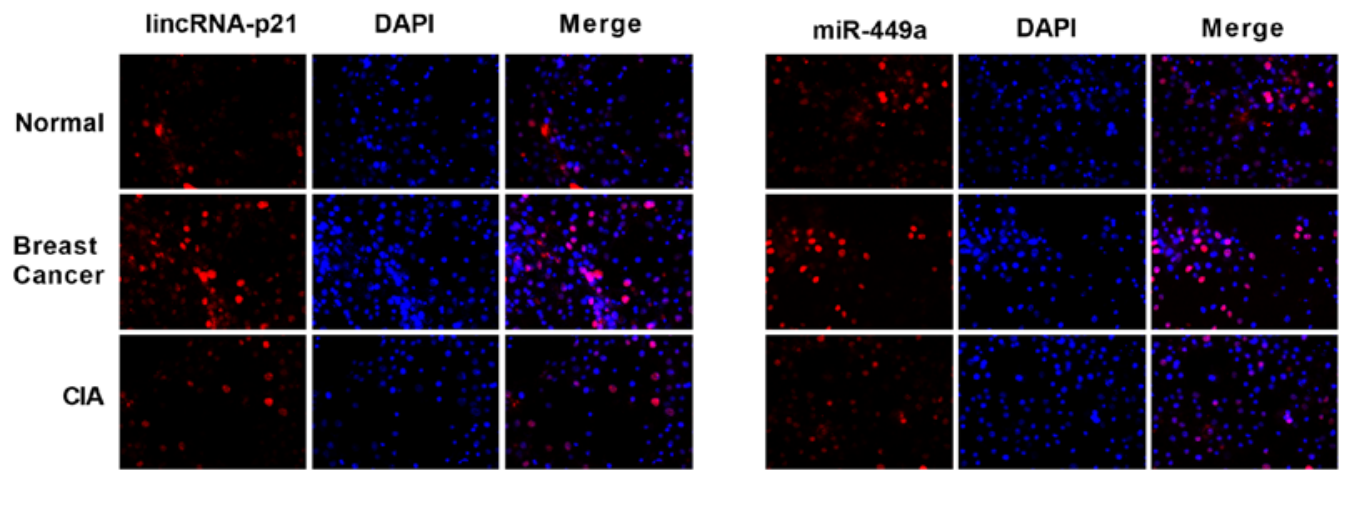

Fig. 1. Altered expression of lincRNA-p21 was observed in both CIA and TNBC group. A Levels of lincRNA-p21 in plasma from CIA $(n=60)$, TNBC $(n=60)$ and C $(n=60)$ groups were measured by RT-qPCR. B FISH results demonstrated that lincRNA-p21 and miR-449a were mainly expressed in the nucleus. All PCR reactions were repeated 3 times, and data were expressed as mean values \pm SD. ${ }^{*} p<0.05$. CIA, cisplatin-induced acute kidney injury; TNBC, triple-negative breast cancer; C, control.

\section{Results}

Altered Expression of lincRNA-p21 Was Observed in Both CIA and TNBC Groups

The expression levels of lincRNA-p21 in plasma of CIA $(n=60)$, TNBC $(n=60)$, and the $\mathrm{C}(n=60)$ groups were measured by RT-qPCR. The expression levels of lincRNA-p21 in the TNBC group were significantly higher than that of CIA and C group (Fig. $1, p<0.05$ ). In addition, expression levels of lincRNA-p21 were also significantly higher in the $\mathrm{C}$ group than that in the TNBC group (Fig. 1a, $p<0.05$ ) Consistent with the qPCR results, the 


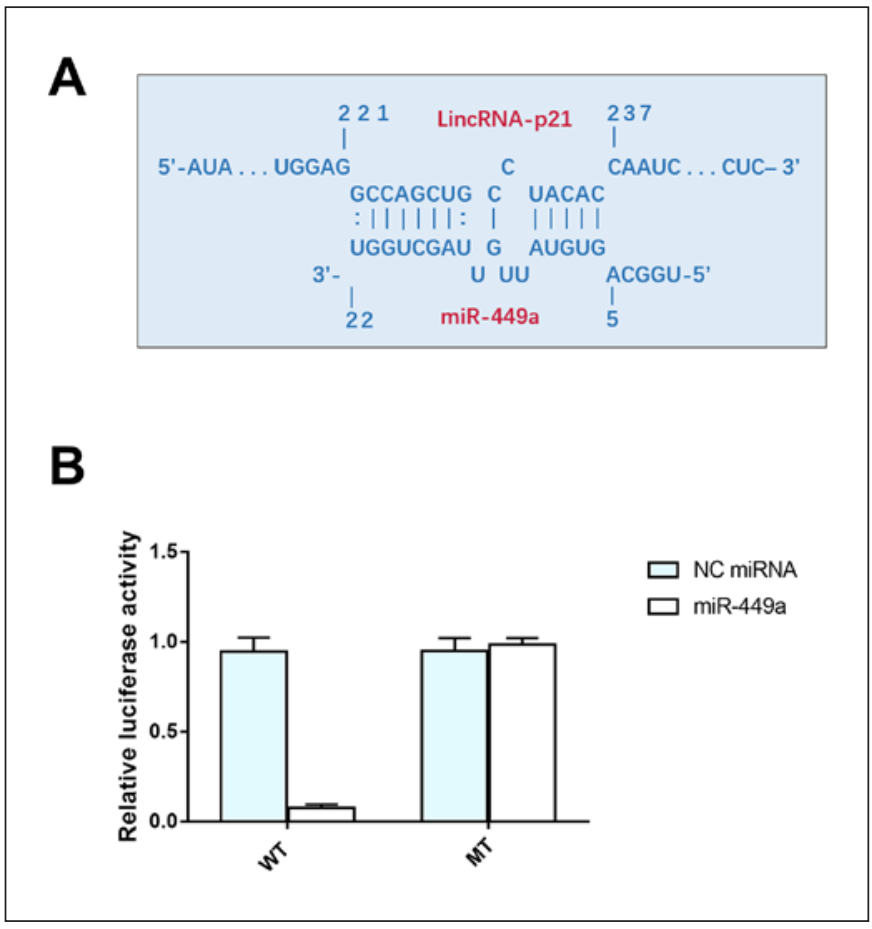

Fig. 2. LincRNA-p 21 and miR-449a can directly interact with each other. A The potential interaction between lincRNA-p21 and miR449a was first predicted by IntaRNA. The interaction between them was further analyzed by performing dual luciferase activity assay by co-transfecting HRPTEpCs with either the combination of lincRNA-p21 vector, mutant lincRNA-p21 vector and miR449a mimic (miR-449a group), or the combination of lincRNAp21 vector and NC miRNA (NC group). B This experiment included 3 biological replicates, and mean values \pm SD were presented and compared. ${ }^{*} p<0.05$. HRPTEpCs, human renal proximal tubular epithelial cells; CIA, cisplatin-induced acute kidney injury.

RNA FISH assay of breast cancer and CIA tissue samples demonstrated that similar expression levels of lincRNAp21 and miR-449a were located in the nucleus (Fig. 1b).

\section{LincRNA-p21 and miR-449a Can Directly Interact with Each Other}

The potential interaction between lincRNA-p21 and miR-449a was first predicted by IntaRNA. It was observed that lincRNA-p21 and miR-449a can form multiple base pairs (Fig. 2a). Dual luciferase activity assay by co-transfecting HRPTEpCs with either the combination of lincRNA-p21 vector and miR-449a mimic (miR-449a group) or the combination of lincRNA-p21 vector and NC miRNA (NC group) was further performed. It showed that the luciferase activity significantly decreased when co-transfected with miR-449a mimics and luciferase re-

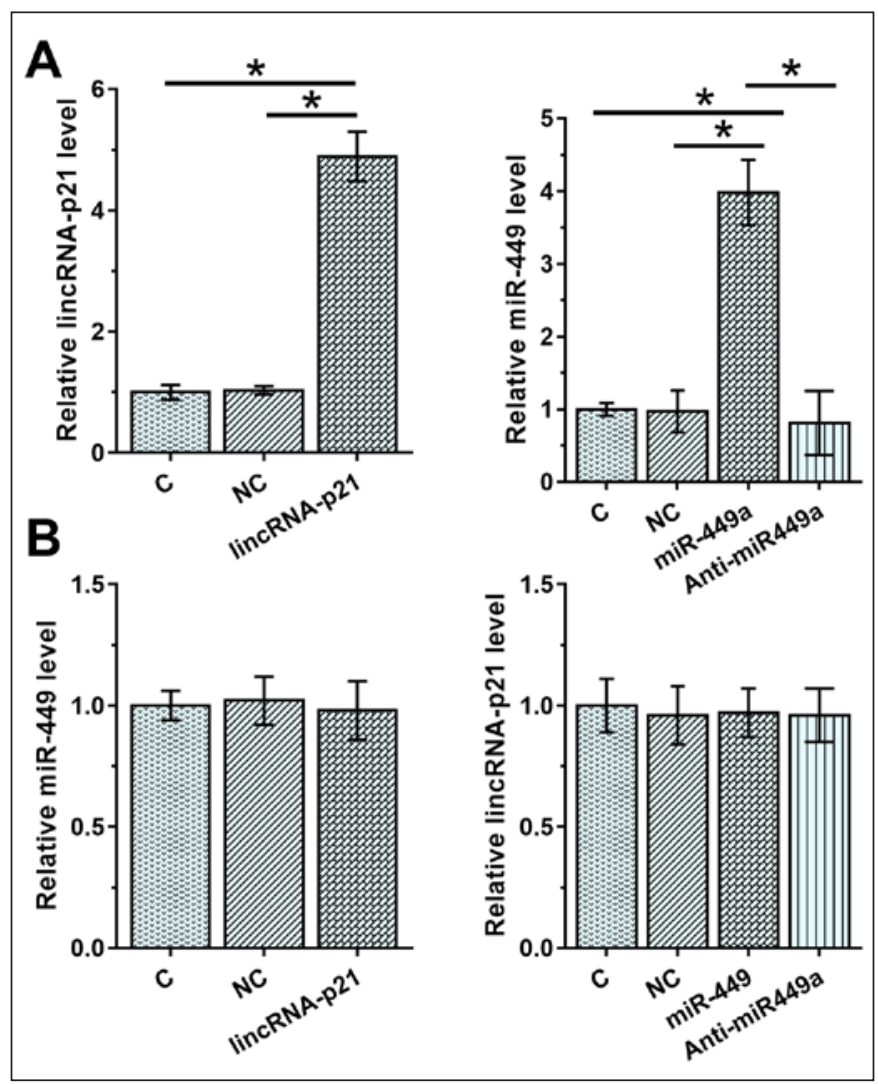

Fig. 3. Overexpression of lincRNA-p21 and miR-449a did not affect the expression of each other. HRPTEpCs were transfected with either lincRNA-p21 expression vector, miR-449a mimic, or antigomiR-449a. A Overexpression of lincRNA-p21 and miR-449a was confirmed by RT-qPCR. B The effects of overexpression of lincRNA-p21 and miR-449a on the expression of each other were analyzed by RT-qPCR. All experiments included 3 biological replicates, and mean values \pm SD were presented and compared. ${ }^{*} p<0.05$. HRPTEpCs, human renal proximal tubular epithelial cells; NC, negative control.

porter. While the key sequence of lincRNA-p21 was mutant, the luciferase activity remained unchanged. Compared to the NC group, the relative luciferase activity was significantly lower in the miR-449a group (Fig. 2b, $p<$ 0.05).

\section{Overexpression of lincRNA-p21 and miR-449a Did}

Not Affect the Expression of Each Other

The relationship between lincRNA-p 21 and miR449a was further evaluated by overexpression experiment. HRPTEpCs were transfected with either lincRNA-p21 expression vector, miR-449a mimic, or antagomiR-449a. Overexpression of lincRNA-p21 and miR-449a was confirmed by RT-qPCR. It showed that 


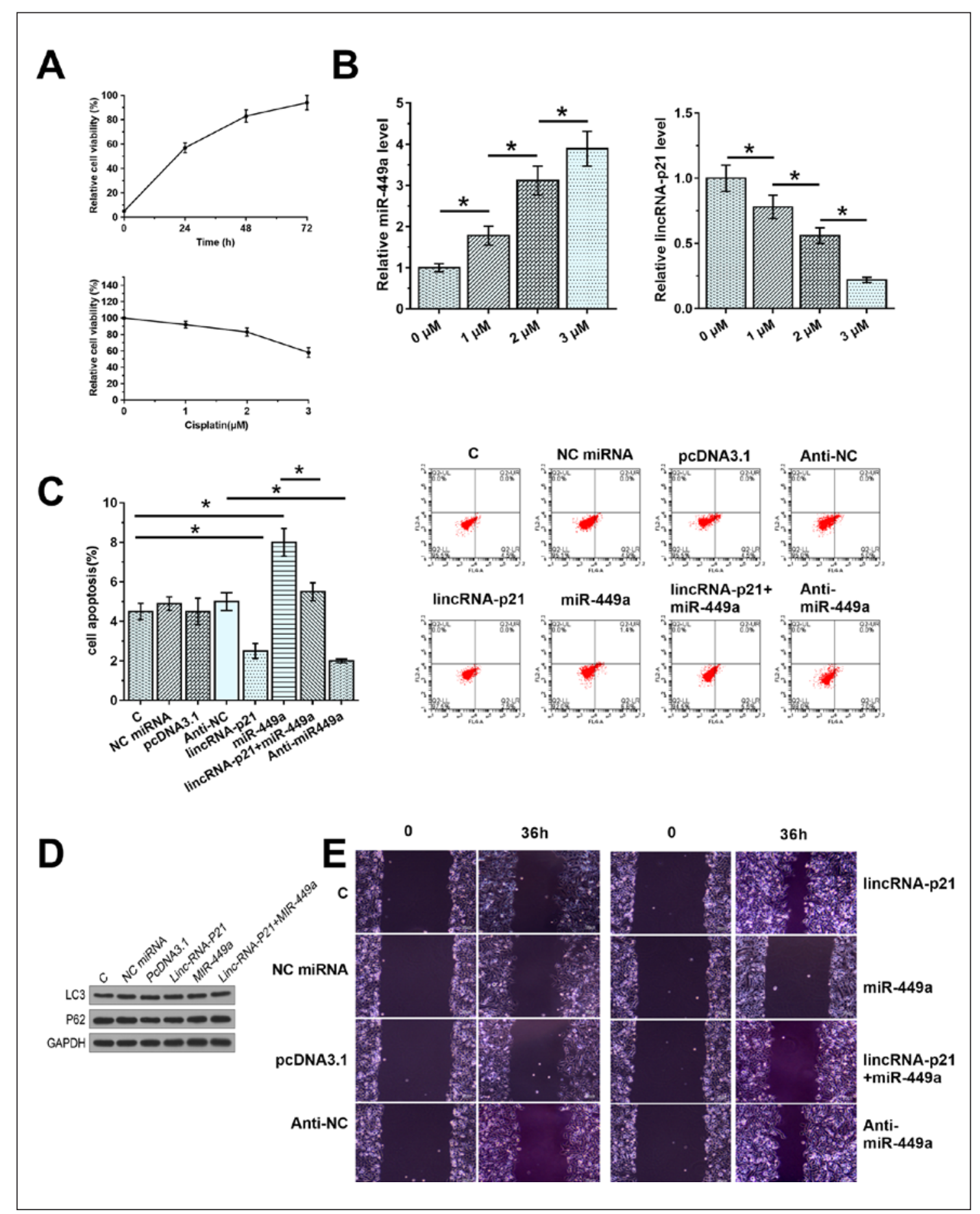

Fig. 4. Overexpression of lincRNA-p21 suppressed cisplatin-induced apoptosis of HRPTEpCs through miR449a. A The time- and dose-dependent cell viability were measured after cisplatin delivered. B HRPTEpCs were treated with 1, 2, or $3 \mu \mathrm{M}$ of cisplatin for $48 \mathrm{~h}$, followed by the measurement of expression levels of lincRNA-p21 and miR-449a by RT-qPCR. C Cell apoptosis assay was performed to analyze the effects of lincRNA-p21 and miR-449a overexpression on the apoptosis of HRPTEpCs. D The expressions of LC3 and p62 were indicated by Western blot. E The healing abilities of lincRNA-p21 and miR-449a overexpression and antagomiR-449a were determined with wound healing assay. All experiments included 3 biological replicates, and mean values \pm SD were presented and compared. ${ }^{*} p<0.05$. HRPTEpCs, human renal proximal tubular epithelial cells; NC, negative control, C, control. 
the expression levels of miR-449a were significantly reduced when transfected with antagomiR-449a (Fig. 3a, $p<0.05)$. Compared to $C$ and NC groups, overexpression of lincRNA-p21 and miR-449a or antagomiR-449a did not affect the expression of each other (Fig. 3b, $p<$ 0.05).

Overexpression of lincRNA-p21 Suppressed CisplatinInduced Apoptosis of HRPTEpCs through miR-449a

The cell damage caused by cisplatin was time- and dose-dependent (Fig. 4a). HRPTEpCs were treated with $0,1,2$, or $3 \mu \mathrm{M}$ of cisplatin for $48 \mathrm{~h}$, and RT-qPCR results showed that cisplatin led to significantly upregulated miR-449a but downregulated lincRNA-p21 (Fig. 4b) in a dose-dependent manner $(p<0.05)$. Cell apoptosis assay was performed to assess the effects of overexpression of lincRNA-p21 and miR-449a on the apoptosis of HRPTEpCs. Compared to the $\mathrm{C}$ group, overexpression of miR449a led to increased apoptotic rate of HRPTEpCs after cisplatin treatment, while this could be reversed by antagomiR-449a (Fig. 4c, $p<0.05$ ). Overexpression of lincRNA-p21 played an opposite role and led to reduced enhancing effects of miR-449a on the cisplatin-induced apoptosis of HRPTEpCs $(p<0.05)$. In addition, Western blot results indicated that the apoptosis was not caused by autophagy due to the nearly unaltered expression levels of marked genes, like LC3 and p62 (Fig. 4d). To determine whether lincRNA-p21 regulated migration and invasion of HRPTEpCs, the wound-healing assay was performed. Overexpression of lincRNA-p21 underwent a quicker closing of scratch wounds than the parallel cell lines containing the empty vector, whereas an opposite result was found when the expression of miR-449a was enhanced (Fig. 4e).

\section{Discussion}

In the past decades, IncRNAs have been reported to act as either therapeutic tools or as molecular biomarkers in cancer $[13,14]$. This study is the first to investigate the involvement of lincRNA-p21 and miR-449a and their interactions in cisplatin-induced CIA in TNBC patients after surgery. We found that the downregulation of lincRNA-p21 may participate in cisplatin-induced CIA in these patients.

The functionality of lincRNA-p21 has been investigated in several types of cancer [15]. For instance, lincRNA-p21 is downregulated in hepatocellular carcinoma and can suppress cell proliferation and promote cell apoptosis by inducing ER stress [15]. In contrast, lncRNA-p21 is overexpressed in prostate cancer, and its elevated expression levels distinguished prostate cancer patients from patients with benign prostate disease, indicating its tumor suppressive role in prostate cancer [16]. Therefore, lincRNA-p21 may have different expression patterns and functions in different types of cancer. The functionality of lincRNA-p21 has not been assessed in TNBC. In this study, we observed higher expression levels of lincRNA-p21 in plasma samples of TNBC patients than in healthy controls, indicating its oncogenic role. However, we measured the expression levels of lincRNAp21 in plasma of TNBC patients after surgical resection, which may affect gene expression. Future studies are needed to measure the expression levels of lincRNA-p21 before treatment.

LincRNA-p2 1 has been proved to promote the development of diabetic nephropathy by sponging miR018b [17]. In this study, we observed the downregulation of plasma lincRNA-p21 in TNBC patients with CIA. Interestingly, the expression levels of lincRNA-p21 in these patients were even lower than those in healthy controls. In addition, in vitro cell experiments showed that overexpression of lincRNA-p21 led to reduced apoptotic rate of HRPTEpCs with cisplatin treatment. Therefore, overexpression of lincRNA-p21 may be a potential therapeutic target for CIA. However, altered expression of lincRNAp21 may induce cancer. It is not rare for some genes that represent a double-edged sword. Due to limited understanding of the functions of lncRNA-p21 in tumor, we postulate that it may have both oncogenic and tumor suppressive roles that are context-dependent in the development and metastasis of HCC [18]. The safety should be further evaluated.

It has been well established that lncRNAs can serve as the endogenous sponge of miRNAs [19]. In this study, we showed that lincRNA-p21 may sponge miR$449 \mathrm{a}$ to reduce its effects on cell apoptosis induced by cisplatin. It was still not clear how lincRNA-p21 regulates miR-449a. As we postulate, there may exist several genes linking between lincRNA-p21 and miR-449a, as well as a downstream target of miR-449a which was the effector controlling the cell proliferation. To resolve this assumption, more studies are still needed in the future. In conclusion, lincRNA-p21 is downregulated with CIA in TNBC patients after surgical resection, and it may sponge miR-449a to suppress cisplatin-induced cell apoptosis. 


\section{Statement of Ethics}

This study passed the review of Ethics Committee of Zaozhuang Municipal Hospital. All participants signed informed consent.

\section{Conflict of Interest Statement}

The authors declare that they have no competing interests.

\section{Funding Sources}

Not applicable.

\section{Authors Contributions}

All authors contributed to data analysis, drafting or revising the article, gave final approval of the version to be published, and agreed to be accountable for all aspects of the work.

\section{References}

1 Kelland L. The resurgence of platinum-based cancer chemotherapy. Nat Rev Cancer. 2007; 7(8):573-84.

2 Dasari S, Tchounwou PB. Cisplatin in cancer therapy: molecular mechanisms of action. Eur J Pharmacol. 2014;740:364-78.

3 Silver DP, Richardson AL, Eklund AC, Wang ZC, Szallasi Z, Li Q, et al. Efficacy of neoadjuvant Cisplatin in triple-negative breast cancer. J Clin Oncol. 2010;28(7):1145-53.

4 Van Acker T, Van Malderen SJ, Van Heerden M, McDuffie JE, Cuyckens F, Vanhaecke F. High-resolution laser ablation-inductively coupled plasma-mass spectrometry imaging of cisplatin-induced nephrotoxic side effects. Anal Chim Acta. 2016;945:23-30.

5 Motwani SS, McMahon GM, Humphreys BD, Partridge AH, Waikar SS, Curhan GC. Development and validation of a risk prediction model for acute kidney injury after the first course of cisplatin. J Clin Oncol. 2018;36(7): 682-8.

6 Malik S, Suchal K, Bhatia J, Gamad N, Dinda AK, Gupta YK, et al. Molecular mechanisms underlying attenuation of cisplatin-induced acute kidney injury by epicatechin gallate. Lab Invest. 2016;96(8):853.
7 Wu HI, Brown JA, Dorie MJ, Lazzeroni L, Brown JM. Genome-wide identification of genes conferring resistance to the anticancer agents cisplatin, oxaliplatin, and mitomycin C. Cancer Res. 2004;64(11):3940-8.

8 Xu H, Robinson GW, Huang J, Lim JY, Zhang $\mathrm{H}$, Bass JK, et al. Common variants in ACYP2 influence susceptibility to cisplatin-induced hearing loss. Nat Genet. 2015;47(3):263-6.

9 Esteller M. Non-coding RNAs in human disease. Nat Rev Genet. 2011;12(12):861-74.

10 Qin W, Xie W, Yang X, Xia N, Yang K. Inhibiting microRNA-449 attenuates cisplatin-induced injury in NRK-52E cells possibly via regulating the SIRT1/P53/BAX pathway. Med Sci Monit. 2016;22:818.

11 Chen S, Liang H, Yang H, Zhou K, Xu L, Liu $\mathrm{J}$, et al. LincRNa-p21: function and mechanism in cancer. Med Oncol. 2017;34(5):98.

12 Mann M, Wright PR, Backofen R. IntaRNA 2.0: enhanced and customizable prediction of RNA-RNA interactions. Nucleic Acids Res. 2017;45(W1):W435-W439.

13 Grimaldi A, Zarone MR, Irace C, Zappavigna S, Lombardi A, Kawasaki H, et al. Non-coding RNAs as a new dawn in tumor diagnosis. Semin Cell Dev Biol. 2018;78:37-50.
14 Cossu AM, Mosca L, Zappavigna S, Misso G, Bocchetti M, De Micco F, et al. Long Noncoding RNAs as Important Biomarkers in Laryngeal Cancer and Other Head and Neck Tumours. Int J Mol Sci. 2019;20(14):3444.

15 Yang N, Fu Y, Zhang H, Sima H, Zhu N, Yang G. LincRNA-p21 activates endoplasmic reticulum stress and inhibits hepatocellular carcinoma. Oncotarget. 2015;6(29):28151-63.

16 Isın M, Uysaler E, Özgür E, Köseoğlu H, Sanlı Ö, Yücel ÖB, et al. Exosomal lncRNA-p21 levels may help to distinguish prostate cancer from benign disease. Front Genet. 2015;6:168.

17 Zhang J, Cao X, Wang S, Aizimaiti M, Xielifu R, Liu J. LincRNA-p21 sponges miR-18b to promote the progression of diabetic nephropathy. Am J Transl Res. 2018;10(5):1481-9.

18 Chen S, Liang H, Yang H, Zhou K, Xu L, Liu $J$, et al. LincRNa-p21: function and mechanism in cancer. Med Oncol. 2017;34(5):98.

19 Paraskevopoulou MD, Hatzigeorgiou AG. Analyzing miRNA-lncRNA interactions. Methods Mol Biol. 2016;1402:271-86. 